\title{
Advances in Cement Research: Referees 2018
}

The following is a list of referees who have reviewed papers for Advances in Cement Research between 1 December 2017 and 30 November 2018 The Institution of Civil Engineers is very grateful for their assistance.

We are continually looking for suitable reviewers for papers submitted to Advances in Cement Research. Papers published in Advances in Cement Research must be submitted to at least two independent referees to judge accuracy, style, impact, importance and interest.

If you are interested in reviewing articles on any topic related to Advances in Cement Research, please submit your name, qualifications or CV and areas of expertise. We are in need of individuals who will agree to review papers in a timely fashion (within 3 to 4 weeks of receipt) and provide confidential feedback to the Editorial Advisory Panel concerning the quality of the paper and any suggested revisions that would be appropriate.

If you are such a person, please contact Cherise Lopes-Baker (tel.: +44 (0)207 665 2453; e-mail: cherise.lopes-baker@icepublishing.com) for more information on the referee process.

\begin{tabular}{|c|c|c|c|}
\hline H Abdelgader & M De Rooij & J Karuppanasamy & R Putra Jaya \\
\hline S Adu-Amankwah & B Demirel & J Katzer & M Quattrone \\
\hline S Agarwal & B Demirel & $\mathrm{XKe}$ & H Rahmani \\
\hline B Akcay & B Dhanya & A Khelidj & B Ramme \\
\hline G Al-Chaar & J Dongell & M Kirgiz & D Revuelta Crespo \\
\hline M Alexander & T Dyer & C Kirk & R Romano \\
\hline J Almeida & D Ectors & M Kolakkadan & F Rubio-Hernández \\
\hline M Alonso & E Eggimann & K Kolovos & M Sadique \\
\hline M Altiner & A El Turki & X Kong & M Safiuddin \\
\hline M Amin & A El-Dieb & R Koodalloor Parasuraman & K Sahoo \\
\hline C Anagnostopoulos & M El-Feky & A Kumar & A Salas \\
\hline Ö Andiç-Çakır & M Emiroglu & W Kunther & A Samadi-Dooki \\
\hline M Antoni & TZ Eng & J Labrincha & M Samchez \\
\hline D Antunes Da Silva & K Erk & G Le Saout & M Sanjuan \\
\hline I Ardelean & J Escalante-Garcia & Y Li & G Sant \\
\hline C Atkins & G Fares & $\mathrm{KLi}$ & I Santacruz \\
\hline S Aydın & P Feng & M Liska & M Santhanam \\
\hline ES Badogiannis & M Florentino Lima Filho & S Liu & A Santra \\
\hline A Bahurudeen & A Fraaij & B Lothenbach & P Sarker \\
\hline M Balonis & W Franke & D Lu & A Segadães \\
\hline P Banfill & E Gallucci & NL Lu & G Selva \\
\hline S Barnett & K Garbev & $\mathrm{H} \mathrm{Ma}$ & V Senthilkumar \\
\hline G Bassioni & P Garces & R Magallanes-Rivera & N Shafiq \\
\hline M Bassuoni & E Garcia-Taengua & H Maraghechi & T Shi \\
\hline M Beazi-Katsioti & S Gauffinet & G Marmol & Z Shi \\
\hline M Ben Haha & D Geddes & S Martinez-Ramirez & C Shi \\
\hline A Bentur & G Geng & B Martín-Pérez & W Shoude \\
\hline AK Bhattacharya & A Ghahremaninezhad & M Medeiros & J Sieber \\
\hline C Bilim & P Ghods & E Menéndez & $N$ Singh \\
\hline S Bishnoi & F Glasser & J Moon & J Skibsted \\
\hline L Black & J Glory & B Mota & L Struble \\
\hline J Buffenbarger & S Goto & M Murugan & T Sui \\
\hline F Bullerjahn & L Guoxin & E Najafi Kani & P Suraneni \\
\hline O Burciaga-Diaz & C Gupta & F G Neunhoeffer & E Tajuelo Rodriguez \\
\hline J Calabria-Holley & C Hall & $\mathrm{S} \mathrm{Ng}$ & Y Tang \\
\hline M Cao & I Harsini & O Ogirigbo & J Tao \\
\hline S Celikten & E Hassan & A Olgun & A Telesca \\
\hline $\mathrm{H}$ Chen & S Horkoss & O Onuaguluchi & P Tennis \\
\hline B Chen & P Hou & T Ozbakkaloglu & J Torrenti \\
\hline J Cheng & $\mathrm{CHu}$ & F Pacheco-Torgal & M Tyrer \\
\hline R Chouhan & M Hubler & M Palacios Arevalo & K Van Breugel \\
\hline N Chousidis & E Irassar & M Palou & S Walling \\
\hline D Chung & A luliia & Z Pan & Q Wang \\
\hline M Cincotto & S Jacobsen & R Pancharathi & Z Wang \\
\hline N Collier & V John & Z Pavlik & S Wang \\
\hline L Csetenyi & S Juradin & A Pisch & N Washburn \\
\hline P Cui & H Justnes & J Plank & R Wasserman \\
\hline J De Brito & S Kandasami & J Provis & X Wei \\
\hline A De La Torre & O Karahan & G Puerta-Falla & M Whittaker \\
\hline
\end{tabular}




$\begin{array}{llll}\text { E Wieland } & \text { L Yan } & \text { M Zhang } & \text { W Zhang } \\ \text { F Winnefeld } & \text { X Yao } & \text { T Zhang } & \text { C Zhou } \\ \text { H Wong } & \text { W Yeih } & \text { Y Zhang } & \text { S Zhou } \\ \text { T Xia } & \text { E Yogarajah } & \text { L Zhang } & \text { X Zhou } \\ \text { J Xu } & \text { C Younsi } & \text { C Zhang } & \text { E Zornoza } \\ \text { B Xu } & \text { M Zajac } & \text { Z Zhang } & \\ \text { P Yan } & \text { Q Zeng } & \text { J Zhang } & \end{array}$

